\title{
TMEM63C, a Potential Novel Target for Albuminuria Development, Is Regulated by MicroRNA-564 and Transforming Growth Factor beta in Human Renal Cells
}

\author{
Miriam Orphal Allan Gillespie Karen Böhme Jana Subrova \\ Andreas Eisenreich Reinhold Kreutz \\ Charité-Universitätsmedizin Berlin, Corporate Member of Freie Universität Berlin, \\ Humboldt-Universität zu Berlin, and Berlin Institute of Health, Institute of Clinical \\ Pharmacology and Toxicology, Berlin, Germany
}

\section{Keywords}

Transmembrane protein 63C - MicroRNA - Podocyte $\cdot$ Transforming growth factor beta .

Epithelial-mesenchymal transition

\begin{abstract}
Introduction: Transmembrane protein (TMEM) $63 \mathrm{C}$ is a member of the TMEM gene family and was recently linked to glomerular filtration barrier function and albuminuria. Its molecular function and expression regulation are largely unknown. Objective: In this study, we set out to characterize the regulating impact of microRNAs (miRNAs) such as miRNA-564 (miR-564) on TMEM63C expression in renal cells. Also, we examined the influence of transforming growth factor beta (TGF- $B$ ) on TMEM63C expression and the potential impact of TMEM63C inhibition on epithelial-mesenchymal transition (EMT) in renal cells and on cell viability in human embryonic kidney 293 cells (HEK 293). Methods: Expression analyses were done using real-time PCR and Western blot. Dual luciferase assay was performed to determine the miRNA-mediated expression control. Cell viability was assessed via trypan blue exclusion staining. Results and Conclusions: MiR-564 reduced TMEM63C expression in HEK 293 and human podocytes (hPC). The treatment of renal cells with TGF- $B$ led to an increased expression of TMEM63C. Moreover, a reduced TMEM63C expression was associated with a changed ratio of EMT marker proteins such as $\alpha$-smooth muscle actin versus E-cadherin in HEK 293 and
\end{abstract}


decreased nephrin expression in hPC. In addition, cell viability was reduced upon inhibition of TMEM63C expression in HEK 293. This study demonstrates first mechanisms involved in TMEM63C expression regulation and a link to EMT in renal cells. (c) 2020 The Author(s).

\section{Introduction}

Podocytes are terminally differentiated and highly specialized glomerular cells with a great importance for the glomerular filtration barrier (GFB) [1]. In this context, nephrin is known as an essential structural protein of the slit diaphragm and is considerably involved in the preservation of glomerular function as well as podocyte viability [2]. The injury or loss of podocytes leads to albuminuria and plays a pivotal role in several glomerular diseases including diabetic and non-diabetic kidney diseases $[3,4]$. While albuminuria itself represents a marker of renal damage, it also has a direct pathogenic effect on renal tissue $[5,6]$. Therefore, albuminuria is discussed as suitable therapeutic target for intervention to slow the progression of CKD [5].

Recently, a study identified transmembrane protein (TMEM) 63C as a novel candidate for albuminuria development [7]. TMEM63C, a member of the TMEM gene family, is expressed in the kidney, but also in many other tissues such as the cerebral cortex and endocrine tissues [7]. Schulz et al. [7] showed a loss of TMEM63C expression in podocytes of patients with focal segmental glomerulosclerosis. In addition, functional studies in zebra fish models suggested that TMEM63C could play an important role in GFB function [7]. Nevertheless, the biological function of TMEM63C and its expression regulation are widely unknown.

MicroRNAs (miRNAs) are short, non-coding RNAs consisting of 20-22 nucleotides, which are known to be influential regulators of post-transcriptional gene expression [8]. The regulation and function of miRNAs are subject of current research. In this context, previous studies described a major influence of miRNAs on kidney diseases [8-10]. For example, miRNA-21 (miR-21), miR-29, and miR-192 were found to play a pathophysiological role in renal fibrosis induced by transforming growth factor beta (TGF- $\beta$ ) [10]. Furthermore, the regulating effect of miRNAs becomes more important in cancer research. In this regard, the impact of several miRNAs, such as miR-21, miR-22, and miR-566, were described in the pathogenesis of renal cell cancer [11-13].

This study sets out to characterize the post-transcriptional regulation of TMEM63C expression by miRNAs including miR-30b and miR-564 in renal cells. Moreover, we analyzed the impact of small interfering RNA (siRNA)-mediated TMEM63C inhibition on cell viability. In addition, the effect of TGF- $\beta$ on TMEM63C regulation and the impact of TMEM63C deficiency on epithelial-mesenchymal transition (EMT) were analyzed in cell culture studies.

\section{Methods}

Cell Culture

Human podocytes (hPC; supplied by Dr. M. Saleem, University of Bristol, Bristol, UK) were cultured and differentiated as described previously [14]. For our investigations, we not only used hPC, but also human embryonic kidney 293 cells (HEK 293) due to its high transfection efficiency and as suitable model to study epithelial characteristics in kidney cells. HEK 293 (a kind gift of Prof. Dr. H. Fechner, Technical University of Berlin, Berlin, Germany) were cultured in DMEM enriched with 10\% fetal bovine serum and 1\% penicillin/streptomycin all provided by Biochrom GmbH, Berlin, Germany. HEK 293 were grown in a humidified incu- 
Kidney and

Blood Pressure

Research

Table 1. The primer sequences of real-time PCR

\begin{tabular}{l|l}
\hline Kidney Blood Press Res 2020;45:850-862 \\
\hline DOI: 10.1159/000508477 & $\begin{array}{l}\text { @ 2020 The Author(s). Published by S. Karger AG, Basel } \\
\text { www.karger.com/kbr }\end{array}$ \\
\hline
\end{tabular}

\begin{tabular}{ll}
\hline Gene & Primer sequences $\left(5^{\prime}->3^{\prime}\right)$ \\
\hline $\begin{array}{l}\text { TMEM63C } \\
\text { Forward } \\
\text { Reverse }\end{array}$ & CAAGCGTGTCCGTAAGGATT \\
\hline GAPDH & ACAATTGGGTCTGCAGCTT \\
Forward & \\
Reverse & GAGTCAACGGATTTGGTCGT \\
\hline Nephrin & GATCTCGCTCCTGGAAGATG \\
Forward & \\
Reverse & ATCCTCTCCATCCTGGTTCC \\
\hline -cadherin & GTCCTGGAGGGACAGAACA \\
Forward & \\
Reverse & ACATTTCCCAACTCCTCTCC \\
\hline$\alpha$-SMA & TCTGTCACCTTCAGCCATC \\
Forward & CGAAGCACAGAGCAAAAGAG \\
Reverse & AGGCATAGAGAGACAGCACCG \\
\hline
\end{tabular}

bator at $37^{\circ} \mathrm{C}$ and with $5 \% \mathrm{CO}_{2}$. Before transfection, HEK 293 and hPC were starved in fetal bovine serum-free DMEM or RPMI 1640, respectively. $200 \mathrm{nM}$ of miR-30b, miR-564, or negative control (miRControl) miRNA mimic was used for miRNA transfection. For siRNA transfection, $200 \mathrm{~nm}$ of TMEM63C siRNAs (siTMEM63C) or scrambled control siRNAs (siControl) were deployed. Transfection was done by using Lipofectamine ${ }^{\mathrm{TM}} 2000$ (Invitrogen $\mathrm{GmbH}$, Karlsruhe, Germany). The inhibitory effect of siTMEM63C was examined on mRNA and protein levels. For stimulation, hPC and HEK 293 were treated with 5 and $10 \mathrm{ng} / \mathrm{mL}$ TGF- $\beta$ (eBioscience; Thermo Fisher Scientific GmbH, Waltham, MA, USA), respectively, for $48 \mathrm{~h}$. Transfection efficiency, determined by Dy547 transfection control $(200 \mathrm{nmol} / \mathrm{L}$; Fisher Scientific - Germany GmbH, Schwerte, Germany), was determined to be $25 \%$ in hPC [14] and 62\% in HEK 293.

\section{Real-Time PCR}

Analyses of mRNA expression were performed as described previously [14]. Specific TaqMan ${ }^{\circledR}$ Gene Expression Assays (Life Technologies GmbH, Darmstadt, Germany) were utilized for determination of TMEM63C, nephrin, $\alpha$-smooth muscle actin ( $\alpha$-SMA), E-cadherin, and glyceraldehyde-3-phosphate dehydrogenase expression, following the manufacturer's instructions. Real-time PCR was accomplished by means of 7500 Fast Real-time PCR System (Applied Biosystems, Carlsbad, CA, USA) using the following conditions: $50^{\circ} \mathrm{C}, 2 \mathrm{~min} ; 95^{\circ} \mathrm{C}$, $20 \mathrm{~s} ; 45$ cycles $95^{\circ} \mathrm{C}, 3 \mathrm{~s} ; 60^{\circ} \mathrm{C}, 30 \mathrm{~s}$. The PCR primers used are shown in Table 1.

\section{Western Blotting}

Western blot analyses were done as described previously [15]. Specific antibodies against TMEM63C (Sigma-Aldrich Chemie GmbH, Munich, Germany) and glyceraldehyde-3-phosphate dehydrogenase (Calbiochem, Darmstadt, Germany) were used for detection. Blots were visualized and quantified by using FUSION FX7 (Peqlab BiotechnologieGmbH, Erlangen, Deutschland) and Gel-Pro Analyser software version 4.0.00.001 (Media Cybernetics, Bethesda, MD, USA).

\section{Dual Luciferase Activity Assay}

For determination of dual luciferase activity, $2 \times 10^{4}$ HEK 293 were treated with miR-30b, miR-564, or miRControl in 96-well plates. Cells were co-transfected with aforementioned 
Kidney and

Blood Pressure

Research

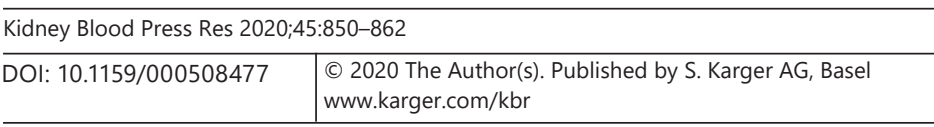

Orphal et al.: TMEM63C Is Regulated by miR-564 and TGF- $\beta$ in Renal Cells

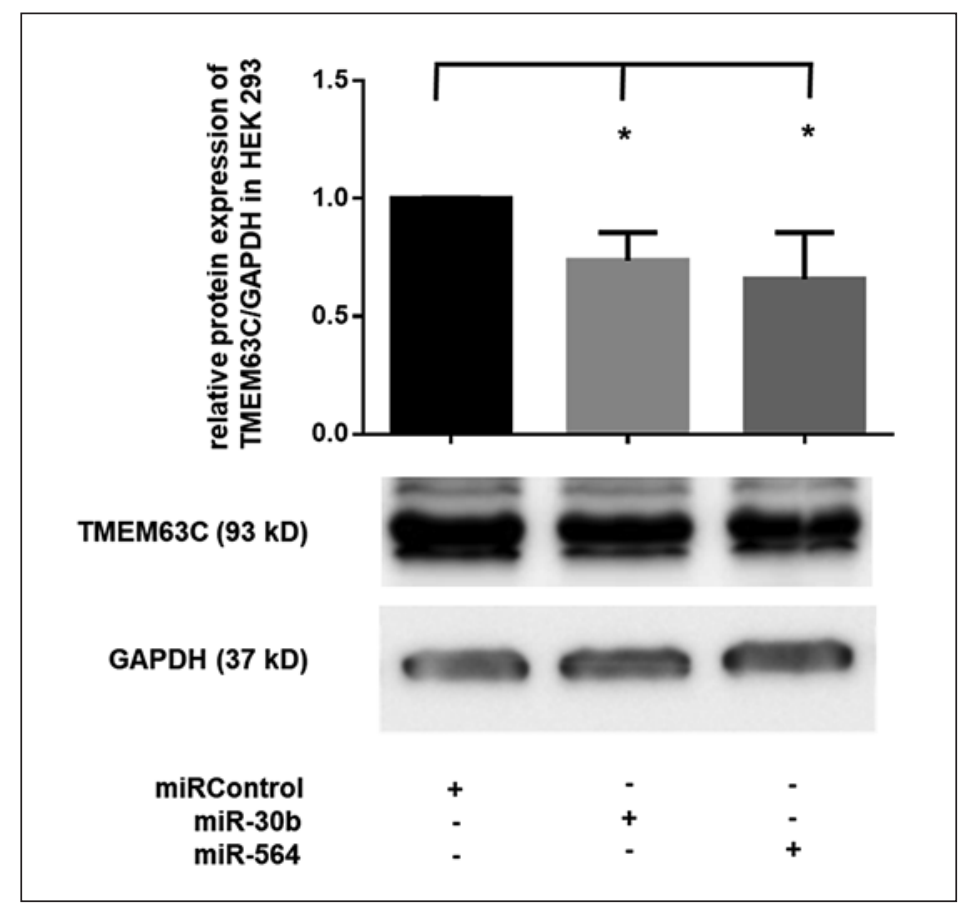

Fig. 1. The impact of miR-30b and miR-564 on TMEM63C protein expression in HEK 293. The protein expression of TMEM63C in HEK 293 transfected with miR-30b, miR-564, or a negative control miRNA mimic (miRControl) is depicted. The expression was analyzed after $48 \mathrm{~h}$. GAPDH was used for normalization. Shown is the mean \pm SEM of at least 3 independent experiments. $\left.{ }^{*}\right) \quad p<0.05$. miR, miRNA; TMEM63C, transmembrane protein 63C; HEK 293, human embryonic kidney cells 293; GAPDH, glyceraldehyde-3-phosphate dehydrogenase.

miRNAs and $200 \mathrm{ng} / \mathrm{mL}$ dual luciferase reporter vector (3'UTR-TMEM63C vec) containing the 3'-untranslated region (3'UTR) of TMEM63C, a reporter vector (3'UTR-TMEM63C-mut vec) containing a mutated miRNA binding site in the $3^{\prime}$ UTR of TMEM63C or a negative control vector (control vec), respectively (provided by GeneCopoeia, Inc., Rockville, MD, USA). After $24 \mathrm{~h}$, dual luciferase activity was analyzed by using a luciferase reporter assay (Promega $\mathrm{GmbH}$, Mannheim, Germany) following the manufacturer's protocol.

\section{Cell Viability Assay}

Cell viability was determined by a trypan blue staining assay. In brief, HEK 293 were seeded in 12-well plates and transfected with $200 \mathrm{nM}$ siTMEM63C or siControl, as described previously. After $48 \mathrm{~h}$, cells were removed from the wells using trypsin-EDTA and subsequently resuspended in fresh DMEM. $10 \mu \mathrm{L}$ of cell suspension was mixed with $90 \mu \mathrm{L}$ of trypan blue solution (Biochrom GmbH, Berlin, Germany). Eventually, living and trypan blue-stained dead cells were counted using a C-Chip Neubauer improved hemocytometer chamber (Carl Roth GmbH \& Co. KG., Karlsruhe, Germany).

\section{Statistical Analysis}

All results were represented as mean \pm standard error the mean (SEM) and analyzed by Student's $t$ test or one-way ANOVA. A probability value $(p)<0.05$ was deemed as significant.

\section{Results}

Impact of Different miRNAs on TMEM63C Expression in HEK 293

First, we performed in silico analysis to identify potential miRNAs modulating TMEM63C expression on post-transcriptional level. In our analysis, we searched relevant databases, such as TargetScanHuman 7.1 (www.targetscan.org), miRDB (www.mirdb.org), and Diana 
(a)

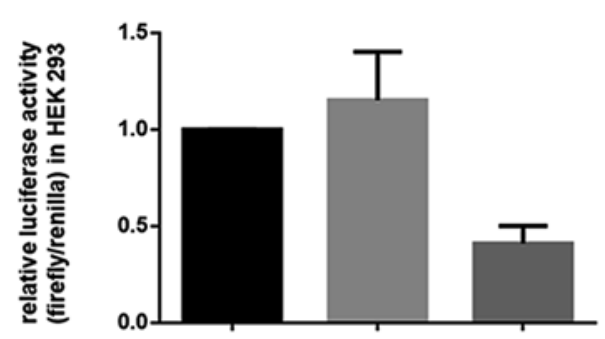

3'UTR-TMEM63C vec miRControl miR-30b miR-564
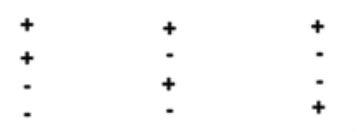

(b)

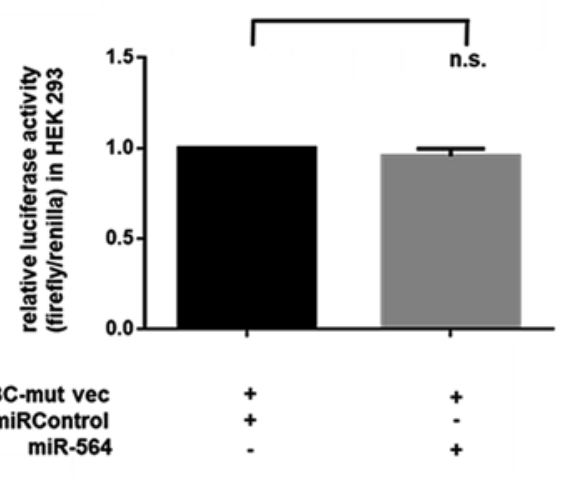

(c)

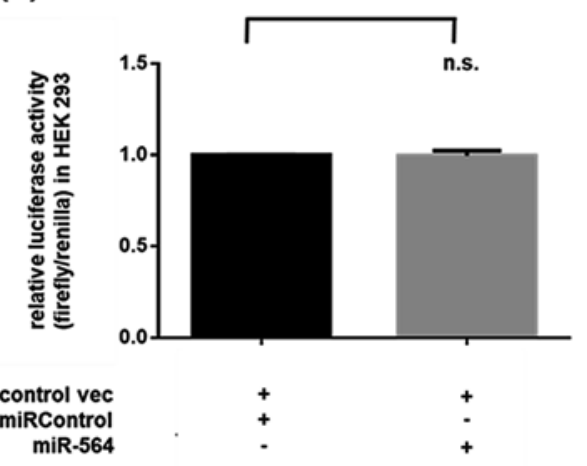

Fig. 2. The influence of miR-30b and miR-564 on luciferase activity in HEK 293. Shown is the luciferase activity in HEK 293 transfected with (a) luciferase reporter construct containing 3'UTR of TMEM63C (3'UTRTMEM63C vec) and co-transfected with miR-30b, miR-564, or a negative control miRNA mimic (miRControl). For control, HEK 293 were co-transfected with (b) control vector (3'UTR-TMEM63C-mut vec), containing a mutated miRNA binding site in the 3'UTR of TMEM63C, and (c) a non-functional control vector (control vec), respectively. Firefly luciferase activity was determined $24 \mathrm{~h}$ after transfection and normalized against renilla luciferase activity. In $\mathbf{a}, \mathbf{b}, \mathbf{c}$, the mean \pm SEM of at least 3 independent experiments is shown. $\left({ }^{* * *}\right) p<$ 0.001. n.s., no significant difference; miR, miRNA; HEK 293, human embryonic kidney cells 293; 3'UTR, 3'-untranslated region; TMEM63C, transmembrane protein 63C.

Tools (www.diana.imis.athena-innovation.gr). All of the abovementioned prediction tools revealed miR-30b and miR-564 as potential candidates for TMEM63C expression regulation via binding to the $3^{\prime}$ UTR of TMEM63C mRNA. To examine the effect of the potential miRNA candidates on TMEM63C expression, we used respective miRNA mimics in our experiments. Next, we determined the influence of the aforementioned miRNAs on protein expression of TMEM63C in HEK 293. In our experiments, miR-30b and miR-564 led to a decreased protein generation of TMEM63C after $48 \mathrm{~h}$ (Fig. 1). 
(a)

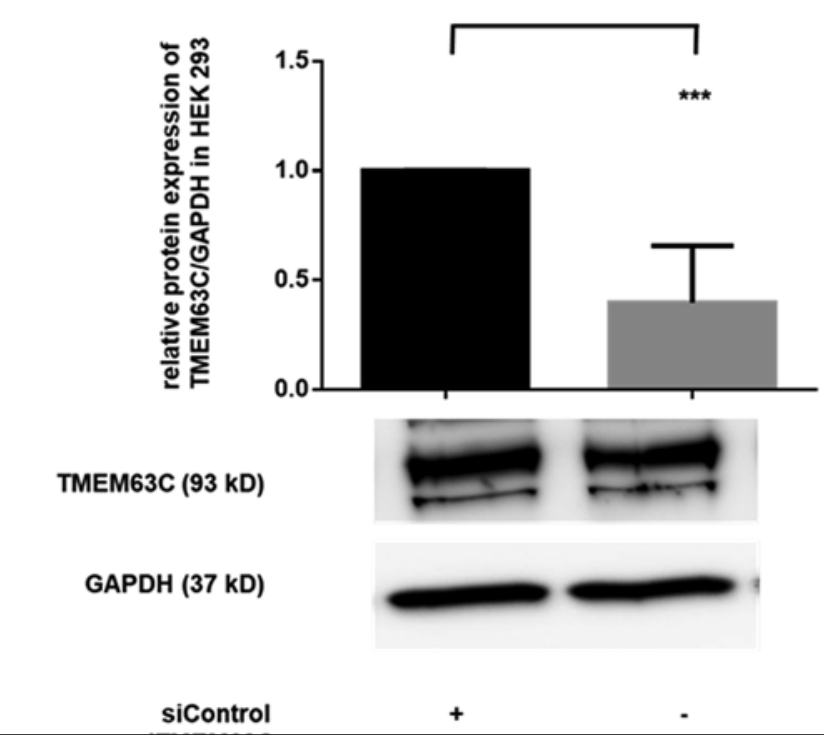

(b)

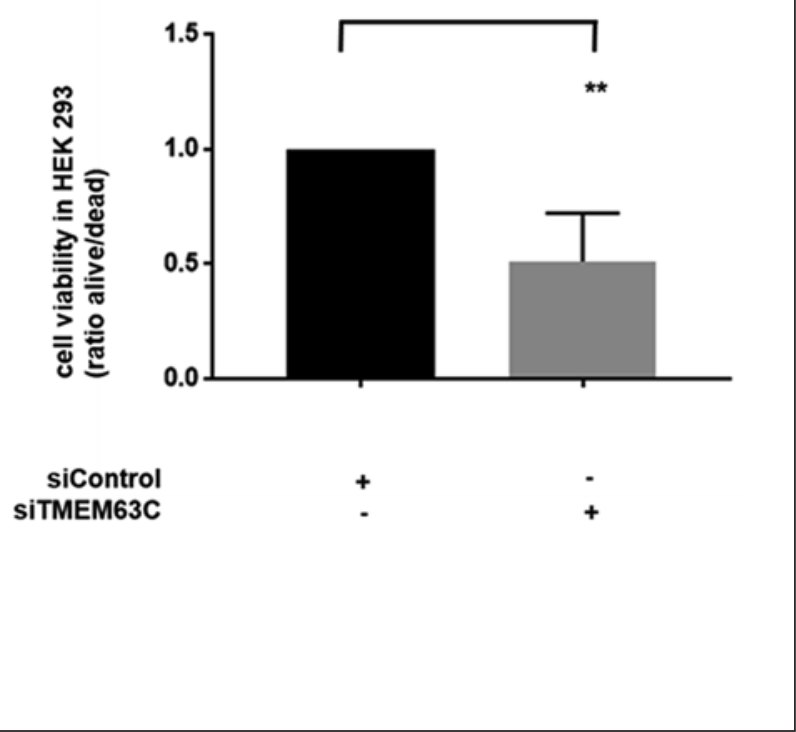

Fig. 3. The impact of TMEM63C inhibition on cell viability in HEK 293. HEK 293 were treated with specific siRNAs against TMEM63C (siTMEM63C) or scrambled control siRNAs (siControl) for $48 \mathrm{~h}$. Shown is the (a) protein expression of TMEM63C and the (b) cell viability of HEK 293. The mean \pm SEM of at least 3 independent experiments is represented. $\left.\left({ }^{* *}\right) p<0.01 ;{ }^{* * *}\right) p<0.001$. TMEM63C, transmembrane protein 63C; HEK 293, human embryonic kidney cells 293; siRNA, small interfering RNA.

\section{Relative Luciferase Activity Measurement in HEK 293}

Compared to controls, co-transfection of cells with the TMEM63C reporter vector and miR-564 led to a reduced luciferase activity, whereas co-transfection with a TMEM63C reporter vector including a mutated 3'UTR binding site and miR-564 exhibited no significant difference in luciferase activity (Fig. 2a, b). In contrast, co-treatment of HEK 293 with the reporter construct and miR-30b had no significant impact on luciferase activity in relation to controls (Fig. 2a).

\section{Cell Viability of HEK 293 Was Decreased by siRNA-Mediated Inhibition of TMEM63C}

After $48 \mathrm{~h}$, we observed a significant reduction of TMEM63C protein expression in siTMEM63C-treated cells (Fig. 3a). Compared to controls, cell viability was also significantly reduced in siTMEM63C-transfected HEK 293 after 48 h (Fig. 3b).

TGF- $\beta$ Stimulation Increased TMEM63C Protein Expression in HEK 293

We analyzed the impact of TGF- $\beta$ stimulation on TMEM63C expression in HEK 293 after $48 \mathrm{~h}$. Compared to controls, treatment of the cells with TGF- $\beta$ increased TMEM63C expression on protein level in a concentration-dependent manner (Fig. 4).

\section{The Ratio of $\alpha$-SMA versus E-Cadherin Was Affected by TMEM63C Expression}

To investigate the impact of TMEM63C on EMT, we transfected HEK 293 with specific siRNAs against TMEM63C for $48 \mathrm{~h}$ and analyzed the ratio of $\alpha$-SMA versus E-cadherin mRNA expression. Compared to controls, the ratio of $\alpha$-SMA versus E-cadherin increased significantly in siTMEM63C-treated cells (Fig. 5). 
Kidney and

Blood Pressure

Research

Fig. 4. The effect of TGF- $\beta$ stimulation on TMEM63C protein expression in HEK 293. HEK 293 were stimulated with 5 or $10 \mathrm{ng} /$ mL TGF- $\beta$ for $48 \mathrm{~h}$. TMEM63C was normalized against GAPDH. The mean \pm SEM of at least 3 independent experiments is shown. $\left({ }^{*}\right) p<$ 0.05 . n.s., no significant difference; TGF- $\beta$, transforming growth factor beta; TMEM63C, transmembrane protein 63C; HEK 293, human embryonic kidney cells 293; GAPDH, glyceraldehyde3-phosphate dehydrogenase.

Fig. 5. The effect of TMEM63C inhibition on $\alpha$-SMA versus E-cadherin. HEK 293 were transfected with inhibitory siRNAs against TMEM63C (siTMEM63C) or nonsense control siRNAs (siControl) for $48 \mathrm{~h}$. Shown is the ratio of mRNA expression of $\alpha$-SMA versus E-cadherin normalized against GAPDH. The mean \pm SEM of at least 3 independent experiments is represented. (***) $p<$ 0.001. TMEM63C, transmembrane protein 63C; $\alpha$-SMA, $\alpha$-smooth muscle actin; HEK 293 , human embryonic kidney cells 293; siRNA, small interfering RNA.

\begin{tabular}{l|l}
\hline Kidney Blood Press Res 2020;45:850-862 \\
\hline DOI: 10.1159/000508477 & $\begin{array}{l}\text { @ } 2020 \text { The Author(s). Published by S. Karger AG, Basel } \\
\text { www.karger.com/kbr }\end{array}$ \\
\hline
\end{tabular}

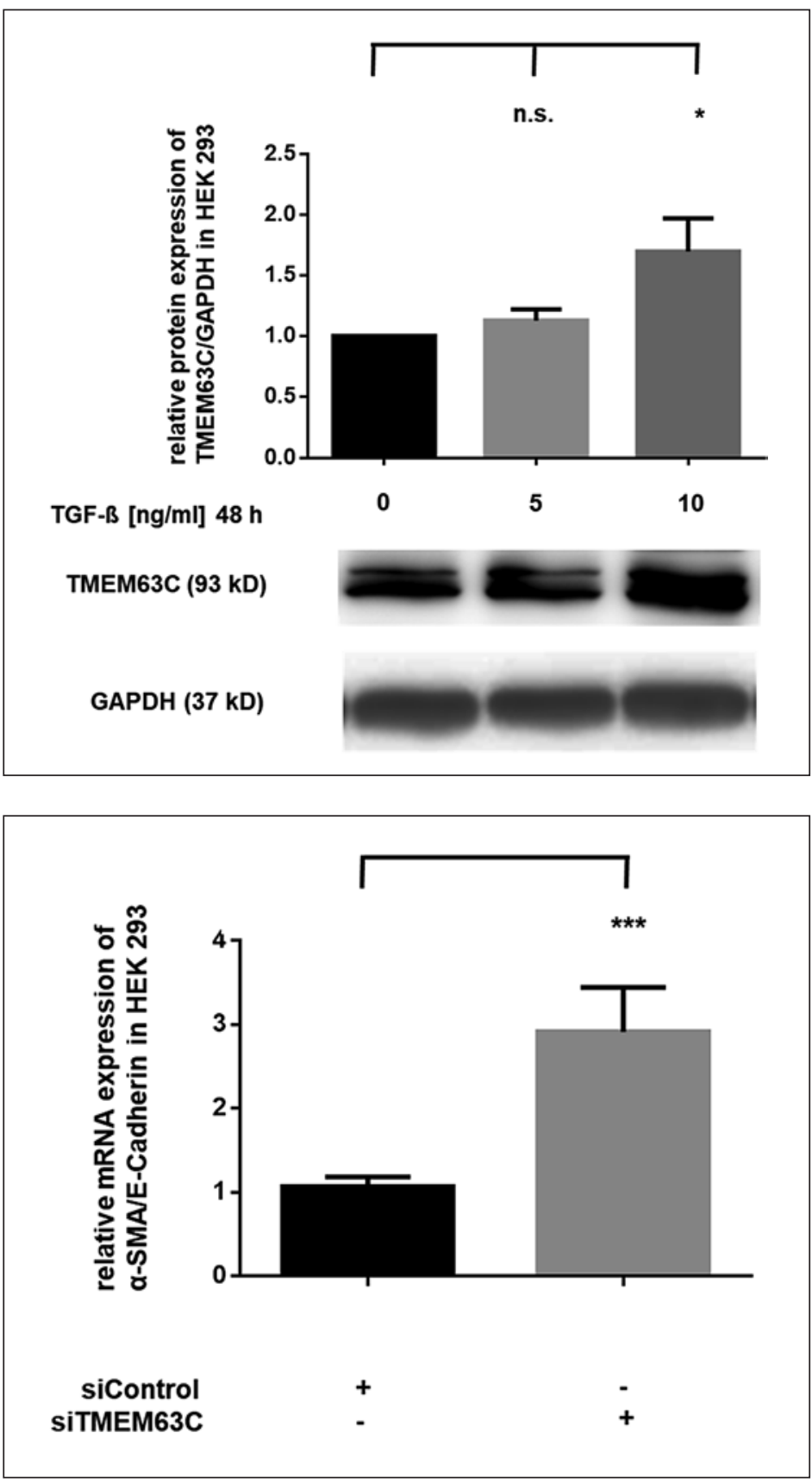

\section{MiR-564 Decreased TMEM63C Expression in hPC}

In addition to the experiments in HEK 293, we analyzed the impact of miR-564 on TMEM63C expression in hPC. Compared to controls, TMEM63C mRNA expression was significantly reduced in miR-564-transfected cells after 48 h (Fig. 6).

\section{TGF- $\beta$ Stimulation Increased TMEM63C Protein Expression in hPC}

Subsequently, we investigated the impact of TGF- $\beta$ on TMEM63C expression in hPC. Compared to controls, the treatment of the cells with 5 or $10 \mathrm{ng} / \mathrm{mL}$ TGF- $\beta$ for $48 \mathrm{~h}$ increased TMEM63C expression on protein level in a concentration-dependent manner (Fig. 7). 
Kidney and

Blood Pressure

Research

Fig. 6. The effect of miR-564 on TMEM63C mRNA expression in hPC. Shown is the expression of TMEM63C mRNA in hPC. Cells were transfected with miR-564 or a negative control miRNA mimic (miRControl). The expression was analyzed after $48 \mathrm{~h}$. GAPDH was used for normalization. The mean \pm SEM of at least 3 independent experiments is shown. (***) $p<0.001$. miR, miRNA; TMEM63C, transmembrane protein $63 \mathrm{C}$; hPC, human podocytes; GAPDH, glyceraldehyde-3-phosphate dehydrogenase.

Fig. 7. The effect of TGF- $\beta$ stimulation on TMEM63C protein expression in hPC. hPC were stimulated with 5 and $10 \mathrm{ng} / \mathrm{mL}$ TGF- $\beta$ for $48 \mathrm{~h}$, respectively. TMEM63C was normalized against GAPDH. The mean \pm SEM of at least 3 independent experiments is shown. $\left(^{*}\right) p<0.05 ;\left({ }^{* * *}\right) p<0.001$. TGF- $\beta$, transforming growth factor beta; TMEM63C, transmembrane protein 63C; hPC, human podocytes; GAPDH, glyceraldehyde-3-phosphate dehydrogenase.

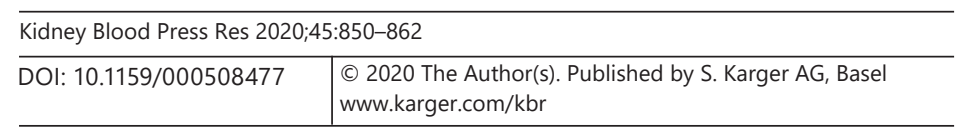

Orphal et al.: TMEM63C Is Regulated by miR-564 and TGF- $\beta$ in Renal Cells
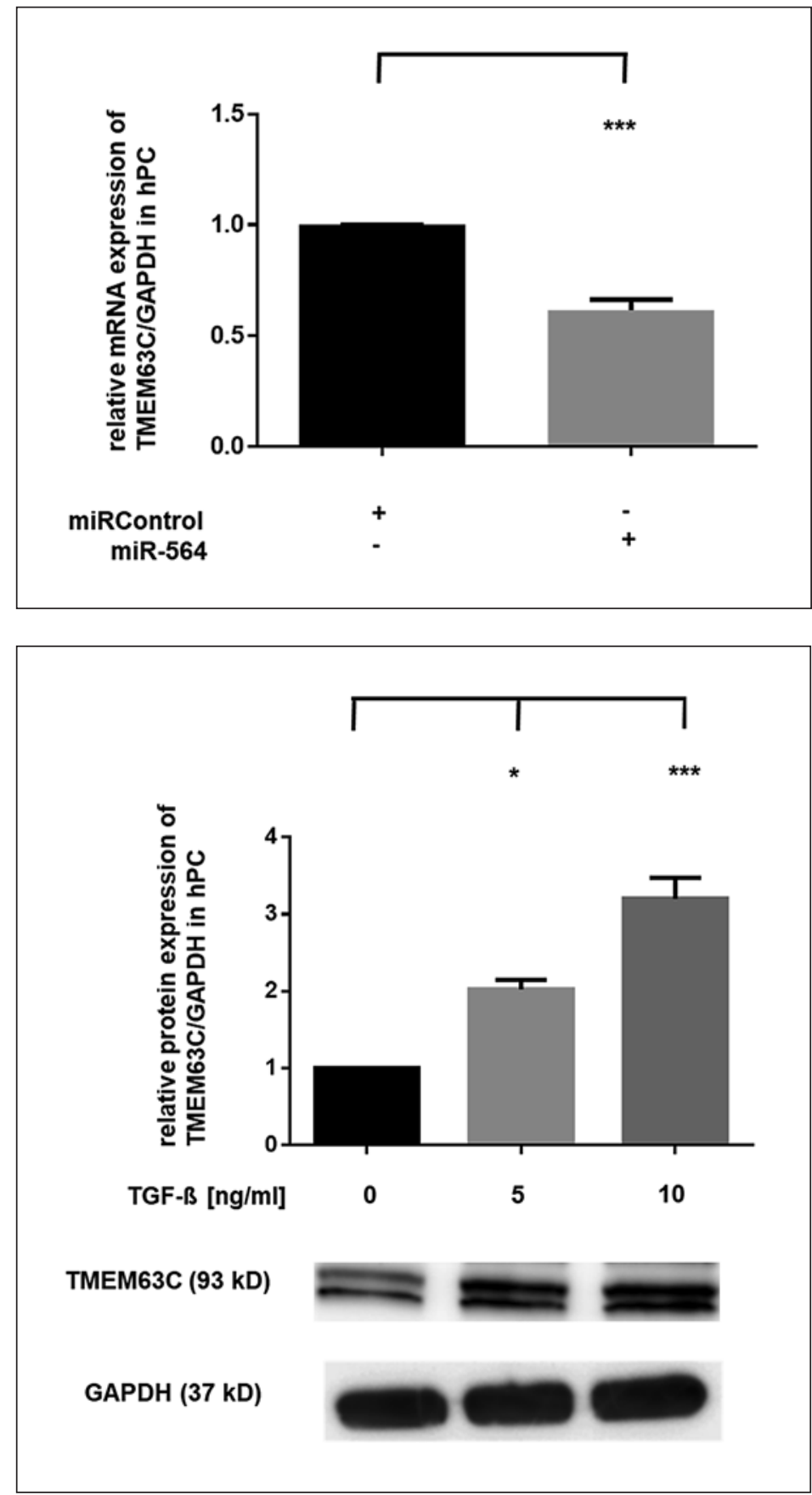

Expression of Nephrin Was Reduced by siRNA-Mediated Inhibition of TMEM63C in hPC

Moreover, we studied the impact of TMEM63C expression on nephrin generation in hPC. Transfection of cells with TMEM63C-specific siRNAs led to a significant reduction of TMEM63C mRNA expression in hPC (Fig. 8a). Compared to controls, siRNA-mediated downregulation of TMEM63C was additionally associated with a significant decrease in nephrin expression on mRNA level after $48 \mathrm{~h}$ (Fig. 8b). 
(a)

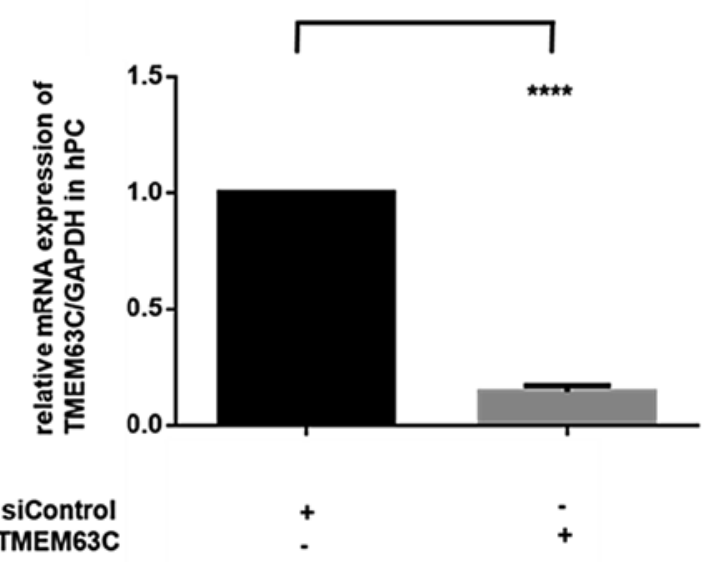

(b)

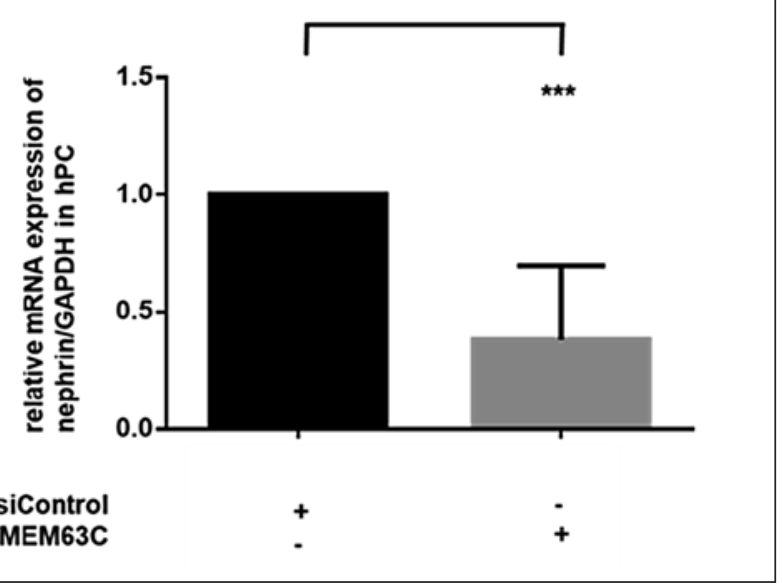

Fig. 8. TMEM63C and nephrin expression in siTMEM63C-treated hPC. hPC were transfected with inhibitory siRNAs against TMEM63C (siTMEM63C) or nonsense control siRNAs (siControl) for $48 \mathrm{~h}$. Shown is the mRNA expression of (a) TMEM63C and (b) nephrin. The mean \pm SEM of at least 3 independent experiments is represented. $\left(^{* * *}\right) p<0.001 ;\left({ }^{* * * *}\right) p<0.0001$. TMEM63C, transmembrane protein 63C; hPC, human podocytes; siRNA, small interfering RNA.

\section{Discussion}

In this study, we identified TMEM63C as a direct target of miR-564. Furthermore, we showed an association between TMEM63C deficiency and reduced cell viability. Moreover, TMEM63C expression was found to be modulated by TGF- $\beta$ stimulation in kidney cells. In addition to previous data [7], our data indicate that TMEM63C could be involved not only in GFB function but also in EMT.

Post-transcriptional Regulation of TMEM63C Expression by miR-564

We investigated the role of miRNAs in the regulation of TMEM63C expression. In a first step, we performed in silico analysis to identify potential miRNAs modulating TMEM63C expression on post-transcriptional level. In silico analysis is based on different computational algorithms, which are using different parameters to predict the probability of a functional miRNA binding site within a given mRNA target [16]. However, the characterization of miRNAmRNA interactions is challenging and difficult to predict [17]. For this reason, several miRNAs were predicted to regulate TMEM63C expression. For our in vitro experiments, we selected miR-30b and miR-564 as very likely candidates for TMEM63C regulation. Both were predicted by at least 3 different prediction tools. In this study, we showed a direct reduction of TMEM63C expression by miR-564 treatment in renal cells, while miR-30b inhibited TMEM63C possibly rather indirectly. Both miRNAs were shown to be expressed in kidney cells [18].

Some miRNAs are already known to be significant participants in renal pathologies, such as miR-21 or miR-192 in kidney fibrosis [10]. In addition, previous studies described a modulating influence of miRNAs, such as miR-21, miR-22, or miR-566, in the genesis or prevention of renal cell cancer [11-13]. So far, miR-564 has not been identified in the context of renal pathologies, but various studies reported on the relevance of miR-564 and its function in different cancer biology. In this regard, miR-564 was demonstrated to induce cell apoptosis and to suppress cell proliferation in osteosarcoma cells by targeting protein kinase B [19]. 
Mutlu et al. [20] showed an inhibitory effect of miR-564 on cell proliferation in breast cancer cells due to suppression of phosphoinositide 3-kinase and mitogen-activated protein kinase. In addition, ectopic expression of miR-564 was described to stop EMT and reduce the migration and invasion of breast cancer cells [20]. In further studies, miR-564 was found to modulate TGF- $\beta$ in different cell types [21, 22]. Jiang et al. [21] showed that miR-564 reduced TGF- $\beta$ expression in glioblastoma cells. In another experimental setting, Xiao and Colleagues [22] presented an upregulation of miR-564 to be associated with an increased TGF- $\beta$ expression in hypertrophic scars. So far, the role of miR-564 in the kidney, and especially in the context of renal pathologies, is unknown. However, miR-564 and its regulative function on TGF- $\beta$ and EMT may not only be important in cancer, but could also be relevant in the modulation of renal fibrosis. In our study, miR-564 suppressed the expression of TMEM63C. For this reason, we investigated the effects of reduced TMEM63C expression on renal cells and studied the impact of TGF- $\beta$ on TMEM63C expression.

\section{Expression Regulation and Functional Effects of TMEM63C in HEK 293}

Schulz et al. [7] showed the importance of TMEM63C expression for the maintenance of GFB in zebra fish models and demonstrated its potential relevance in patients with focal segmental glomerulosclerosis in translational studies. However, until now, the expression regulation of TMEM63C is widely unexplored. In our experiments, we used HEK 293 - due to its high transfection efficiency - to examine the expression regulation and impact of TMEM63C. The recent report already showed impaired cell viability due to suppression of TMEM63C in hPC [7]. In our study, we found downregulation of TMEM63C to be associated with reduced viability also in other renal cells (HEK 293). These results suggest that TMEM63C may have a beneficial effect on renal cell survival. Next, we analyzed the impact of TGF- $\beta$ on TMEM63C expression. TGF- $\beta$ is known as regulator and initiator of EMT and has been described as key mediator of glomerular and tubulointerstitial pathobiology in CKD $[23,24]$. In CKD, TGF- $\beta$ led to tubulointerstitial fibrosis and dysfunction of podocytes [25]. Previous studies showed that miR-564 is involved in the regulation of TGF- $\beta[21,22]$. In this study, we found TGF- $\beta$ to affect TMEM63C expression in a concentration-dependent manner. Further, we observed an inhibiting effect of miR-564 on TMEM63C generation. For this reason, we also studied the potential role of TMEM63C in EMT. In EMT, cells lose progressively their epithelial characteristics and acquire markers of mesenchymal phenotype [26]. Therefore, the ratio of epithelial and mesenchymal marker proteins is changed [27]. Typical epithelial markers are E-cadherin and fibronectin, whereas typical mesenchymal proteins are $\mathrm{N}$-cadherin, $\alpha$-SMA, and vimentin [27]. HEK 293 were shown to express both epithelial and mesenchymal marker proteins [28]. Therefore, we used these cells as suitable model to study the change of epithelial characteristics by altered TMEM63C expression. We found reduced TMEM63C expression to be associated with an increased ratio of $\alpha$-SMA versus E-cadherin in HEK 293. This changing ratio of $\alpha$-SMA versus E-cadherin is discussed as an indicator for tubular EMT [29]. However, the precise role of TMEM63C in EMT of renal tubular epithelial cells remains uncertain and should be investigated in further experiments. In conclusion, we found siTMEM63C to reduce cell viability in HEK 293. In addition, we showed an association between reduced TMEM63C expression and a changing expression of proteins that are involved in EMT. Therefore, the increased expression of TMEM63C by TGF- $\beta$ might reflect a potential protective mechanism of TMEM63C in renal cells under pathophysiological conditions, such as the development of renal fibrosis.

\section{Expression Regulation and Functional Effects of TMEM63C in hPC}

Similar to HEK 293, TGF- $\beta$ treatment increased TMEM63C expression in a concentrationdependent manner in hPC. Enhanced TGF- $\beta$ concentration is associated with podocyte apoptosis and detachment from the glomerular basement membrane, EMT, and the development 
of glomerulosclerosis [30]. For EMT studies in podocytes, it is important to consider that these cells have been described as atypical epithelial cells with a different expression of epithelial and mesenchymal proteins [31]. EMT in podocytes, also referred to as "podocyte disease transformation," is associated with a decrease in proteins such as podocin, nephrin, and P-cadherin and an increase in $\alpha$-SMA and N-cadherin [31, 32]. Moreover, Ghiggeri et al. [33] indicated that nephrin elimination may trigger EMT in hPC. We showed that reduced TMEM63C expression is associated with decreased generation of nephrin in hPC. These data support our suspicion that TMEM63C may play a potential role in modulation of EMT in renal cells. Further, nephrin is known as an essential structural protein of the slit diaphragm and is pivotal for the preservation of GFB function as well as podocyte viability [2]. Langham et al. [34] also showed reduced nephrin expression to be associated with proteinuria in relevant kidney diseases such as diabetic nephropathy. We found reduced nephrin expression by inhibiting TMEM63C. For this reason, the current data support our previous observations linking TMEM63C to the development of albuminuria [7]. Altogether, our results demonstrated that TMEM63C is regulated by miR-564 and TGF- $\beta$ in hPC and affects the expression of nephrin and could therefore influence the preservation of renal filter function.

\section{Conclusion}

In this study, we identified TMEM63C as a direct target of miR-564 in human renal cells. Moreover, the current study demonstrated that TMEM63C is regulated by TGF- $\beta$ and could be involved in EMT. However, the effects of TMEM63C on EMT have not yet been clarified and should be investigated in further experiments. These experiments may include in vivo studies or at least studies in primary kidney cells, since experiments in immortalized cell lines, although important for mechanistic studies, should be viewed with some caution due to a potential permissive oncogenic phenotype for EMT studies [35]. Moreover, the role of EMT per se in the context of renal fibrosis has been questioned [36] and has been a matter of controversy [37]. Nevertheless, more recent studies indicated that at least partial EMT may be sufficient to promote renal fibrosis [38,39] although evidence for human CKD is scant [37].

Our current results also support recently reported findings linking TMEM63Cexpression to GFB function and albuminuria [7]. In the latter study, downregulation of TMEM63C resulted in albuminuria in zebra fish models and reduced cell viability of hPC, indicating a potential protective effect of TMEM63C for renal filter function [7]. In line with these findings, the current study showed siTMEM63C to reduce nephrin, a pivotal protein for the preservation of GFB function as well as podocyte viability [2]. Moreover, siRNA-mediated downregulation of TMEM63C led to a reduced cell viability of HEK 293. On the other hand, downregulation ofTMEM63CinHEK293was linkedtoEMTbyincreasingthe $\alpha$-SMA/E-cadherin ratio and was thus associated with the activation of pro-fibrotic mechanisms [37]. In this regard, our finding demonstrating an upregulation of TMEM63C by TGF- $\beta$, which is a major driver of renal fibrosis, may reflect a counter-balancing mechanistic link between TMEM63C and TGF- $\beta$. Taken together, our data indicate that TMEM63C is not only linked to GFB function but may also be involved in EMT control. Therefore, TMEM63C is a novel functional candidate with potential impact for the development and progression of CKD.

\section{Acknowledgements}

We thank Claudia Plum, Petra Karsten, Sarah Podlech, Dr. Anja Brehm, and Marie Käbel for experimental and technical support. 


\section{Conflict of Interest Statement}

The authors have no conflicts of interest to declare.

\section{Funding Sources}

This study was supported by the DFG (German Research Foundation) - Project number 394046635-SFG 1365 and the Open Access Publication Funds of Charité - Universitätsmedizin Berlin.

\section{Author Contributions}

M.O., A.G., J.S., K.B., and A.E. carried out the cell culture studies. K.B., M.O., and A.E. participated in the PCR tests. M.O. and A.E. performed the statistical analysis. M.O., A.E., and R.K. conceived the study, participated in its design and coordination, and drafted the manuscript. A.G., J.S., and K.B. revised the manuscript critically. All authors read and approved the final manuscript. All authors agree to be accountable for all aspects of the work and to ensure that questions relating to the accuracy or integrity of any part of the work have been appropriately investigated and resolved.

TMEM63C, transmembrane protein 63C; GAPDH, glyceraldehyde-3-phosphate dehydrogenase; $\alpha$-SMA, $\alpha$-smooth muscle actin.

\section{References}

1 Reiser J, Sever S. Podocyte biology and pathogenesis of kidney disease. Annu Rev Med. 2013;64:357-66.

2 Li X, Chuang PY, D’Agati VD, Dai Y, Yacoub R, Fu J, et al. Nephrin preserves podocyte viability and glomerular structure and function in adult kidneys. J Am Soc Nephrol. 2015;26(10):2361-77.

3 Podgorski P, Konieczny A, Lis L, Witkiewicz W, Hruby Z. Glomerular podocytes in diabetic renal disease. Adv Clin Exp Med. 2019;28(12):1711-5.

4 Brinkkoetter PT, Ising C, Benzing T. The role of the podocyte in albumin filtration. Nat Rev Nephrol. 2013;9(6): 328-36.

5 Lambers Heerspink HJ, Gansevoort RT. Albuminuria is an appropriate therapeutic target in patients with CKD: the pro view. Clin J Am Soc Nephrol. 2015;10(6):1079-88.

6 Abbate M, Zoja C, Remuzzi G. How does proteinuria cause progressive renal damage? J Am Soc Nephrol. 2006; 17(11):2974-84.

7 Schulz A, Müller NV, van de Lest NA, Eisenreich A, Schmidbauer M, Barysenka A, et al. Analysis of the genomic architecture of a complex trait locus in hypertensive rat models links Tmem63c to kidney damage. eLife. 2019; 8:e42068.

8 Trionfini P, Benigni A. MicroRNAs as master regulators of glomerular function in health and disease. J Am Soc Nephrol. 2017;28(6):1686-96.

9 Trionfini P, Benigni A, Remuzzi G. MicroRNAs in kidney physiology and disease. Nat Rev Nephrol. 2014;11(1): 23.

10 Chung AC, Lan HY. MicroRNAs in renal fibrosis. Front Physiol. 2015;6:50.

11 Fan B, Jin Y, Zhang H, Zhao R, Sun M, Sun M, et al. MicroRNA21 contributes to renal cell carcinoma cell invasiveness and angiogenesis via the PDCD4/cJun (AP1) signalling pathway. Int J Oncol. 2020 Jan;56(1):178-92.

12 Gong X, Zhao H, Saar M, Peehl DM, Brooks JD. miR-22 regulates invasion, gene expression and predicts overall survival in patients with clear cell renal cell carcinoma. Kidney Cancer. 2019;3(2):119-32.

13 Pan X, Quan J, Li Z, Zhao L, Zhou L, Jinling X, et al. miR-566 functions as an oncogene and a potential biomarker for prognosis in renal cell carcinoma. Biomed Pharmacother. 2018;102:718-27.

14 Eisenreich A, Langer S, Herlan L, Kreutz R. Regulation of podoplanin expression by microRNA-29b associates with its antiapoptotic effect in angiotensin II-induced injury of human podocytes. J Hypertens. 2016;34(2): 323-31.

15 Leppert U, Gillespie A, Orphal M, Böhme K, Plum C, Nagorsen K, et al. The impact of $\alpha$-lipoic acid on cell viability and expression of nephrin and ZNF580 in normal human podocytes. Eur J Pharmacol. 2017;810:1-8. 
16 Kuhn DE, Martin MM, Feldman DS, Terry AV Jr., Nuovo GJ, Elton TS. Experimental validation of miRNA targets. Methods. 2008;44(1):47-54.

17 Riffo-Campos ÁL, Riquelme I, Brebi-Mieville P. Tools for sequence-based miRNA target prediction: what to choose? Int J Mol Sci. 2016;17(12):1987.

18 Argyropoulos C, Wang K, Bernardo J, Ellis D, Orchard T, Galas D, et al. Urinary microRNA profiling predicts the development of microalbuminuria in patients with type 1 diabetes. J Clin Med. 2015;4(7):1498-517.

19 Ru N, Zhang F, Liang J, Du Y, Wu W, Wang F, et al. MiR-564 is down-regulated in osteosarcoma and inhibits the proliferation of osteosarcoma cells via targeting Akt. Gene. 2018;645:163-9.

20 Mutlu M, Saatci Ö, Ansari SA, Yurdusev E, Shehwana H, Konu Ö, et al. miR-564 acts as a dual inhibitor of PI3K and MAPK signaling networks and inhibits proliferation and invasion in breast cancer. Sci Rep. 2016;6:32541.

21 Jiang C, Shen F, Du J, Hu Z, Li X, Su J, et al. MicroRNA-564 is downregulated in glioblastoma and inhibited proliferation and invasion of glioblastoma cells by targeting TGF- $\beta 1$. Oncotarget. 2016;7(35):56200-8.

22 Xiao L, Tang T, Huang Y, Guo J. MiR-564 promotes hypertrophic scar via up-regulating TGF- $\beta 1$. G Ital Dermatol Venereol. 2019 Apr;154(2):186-91.

23 Bottinger EP, Bitzer M. TGF-beta signaling in renal disease. J Am Soc Nephrol. 2002;13(10):2600-10.

24 Loeffler I, Wolf G. Transforming growth factor- $\beta$ and the progression of renal disease. Nephrol Dial Transplant. 2014;29(Suppl 1):i37-45.

25 Gewin L, Zent R. How does TGF- $\beta$ mediate tubulointerstitial fibrosis? Semin Nephrol. 2012;32(3):228-35.

26 Seccia T, Caroccia B, Piazza M, Rossi GP. The key role of epithelial to mesenchymal transition (EMT) in hypertensive kidney disease. Int J Mol Sci. 2019;20(14):3567.

27 Moustakas A, Heldin CH. Mechanisms of TGF $\beta$-induced epithelial-mesenchymal transition. J Clin Med. 2016; $5(7): 63$.

28 Inada M, Izawa G, Kobayashi W, Ozawa M. 293 cells express both epithelial as well as mesenchymal cell adhesion molecules. Int J Mol Med. 2016;37(6):1521-7.

29 Du T, Zou X, Cheng J, Wu S, Zhong L, Ju G, et al. Human Wharton's jelly-derived mesenchymal stromal cells reduce renal fibrosis through induction of native and foreign hepatocyte growth factor synthesis in injured tubular epithelial cells. Stem Cell Res Ther. 2013;4(3):59.

30 Lee HS. Mechanisms and consequences of TGF- $\beta$ overexpression by podocytes in progressive podocyte disease. Cell Tissue Res. 2012;347(1):129-40.

31 May CJ, Saleem M, Welsh GI. Podocyte dedifferentiation: a specialized process for a specialized cell. Front Endocrinol. 2014;5:148.

32 Ying Q, Wu G. Molecular mechanisms involved in podocyte EMT and concomitant diabetic kidney diseases: an update. Ren Fail. 2017;39(1):474-83.

33 Ghiggeri GM, Gigante M, Di Donato A. Constitutional nephrin deficiency in conditionally immortalized human podocytes induced epithelial-mesenchymal transition, supported by beta-catenin/NF-kappa B activation: a consequence of cell junction impairment? Inter J Nephrol. 2013;2013:457490.

34 Langham RG, Kelly DJ, Cox AJ, Thomson NM, Holthöfer H, Zaoui P, et al. Proteinuria and the expression of the podocyte slit diaphragm protein, nephrin, in diabetic nephropathy: effects of angiotensin converting enzyme inhibition. Diabetologia. 2002;45(11):1572-6.

35 Wang Y, Chen S, Yan Z, Pei M. A prospect of cell immortalization combined with matrix microenvironmental optimization strategy for tissue engineering and regeneration. Cell Biosci. 2019;9(1):7.

36 Humphreys BD, Lin SL, Kobayashi A, Hudson TE, Nowlin BT, Bonventre JV, et al. Fate tracing reveals the pericyte and not epithelial origin of myofibroblasts in kidney fibrosis. Am J Pathol. 2010;176(1):85-97.

37 Liu BC, Tang TT, Lv LL, Lan HY. Renal tubule injury: a driving force toward chronic kidney disease. Kidney Int. 2018;93(3):568-79.

38 Lovisa S, LeBleu VS, Tampe B, Sugimoto H, Vadnagara K, Carstens JL, et al. Epithelial-to-mesenchymal transition induces cell cycle arrest and parenchymal damage in renal fibrosis. Nat Med. 2015;21(9):998-1009.

39 Grande MT, Sánchez-Laorden B, López-Blau C, De Frutos CA, Boutet A, Arévalo M, et al. Snail1-induced partial epithelial-to-mesenchymal transition drives renal fibrosis in mice and can be targeted to reverse established disease. Nat Med. 2015;21(9):989-97. 